\title{
Evaluación constructiva-cronométrica como herramienta para evaluar el aprendizaje en línea y presencial
}

\author{
Guadalupe Elizabeth Morales Martínez \\ Investigadora del Laboratorio de Ciencia Cognitiva del Instituto de Investigaciones sobre la \\ Universidad y la Educación de la Universidad Nacional Autónoma de México \\ gemoramar@hotmail.com \\ Rafael Manuel López Pérez \\ Presidente de la Fundación Universitaria Behavior \& Law \\ rlopez@behaviorandlaw.com \\ Ángel García Collantes \\ Profesor del grado de Criminología de la Universidad a Distancia de Madrid, UDIMA \\ angel.garcia.c@udima.es \\ Ernesto Octavio López Ramírez (†) \\ Investigador del Laboratorio de Ciencia Cognitiva de la Facultad de \\ Psicología de la Universidad Autónoma de Nuevo León
}

\section{Extracto}

Se presenta una forma innovadora de aproximar la evaluación del aprendizaje desde una visión cognitiva. Para ello, se ejemplifica el uso de un modelo de medición constructiva-cronométrica del aprendizaje a través de varios dominios del conocimiento y se ilustra la forma en que este tipo de evaluación proporciona diversos indicadores de organización y estructura del esquema de conocimiento que el estudiante presenta antes y después de haber cursado una materia. La evidencia obtenida sugiere que el modelo de evaluación aquí propuesto es efectivo en la medición cognitiva del aprendizaje académico, lo que indica que la implementación de herramientas de evaluación de la ciencia cognitiva en el campo de la educación puede ser muy útil para ampliar nuestra comprensión sobre el proceso de adquisición de conocimiento. Estas y otras implicaciones para la evaluación del aprendizaje de los estudiantes se discuten en el presente trabajo.

Palabras clave: evaluación digital del aprendizaje; ciencia cognitiva; simulaciones de esquema de conocimiento; facilitación semántica; redes semánticas naturales.

Fecha de entrada: 10-06-2019 / Fecha de revisión: 20-07-2019 / Fecha de aceptación: 29-08-2019

Cómo citar: Morales Martínez, G. E., López Pérez, R. M., García Collantes, Á. y López Ramírez, E. O. (2020). Evaluación constructiva-cronométrica como herramienta para evaluar el aprendizaje en línea y presencial. Tecnología, Ciencia y Educación, 15, 105-124. 


\title{
Constructive-chronometric evaluation as a tool to evaluate online and face-to-face learning
}

\author{
Guadalupe Elizabeth Morales Martínez \\ Rafael Manuel López Pérez \\ Ángel García Collantes \\ Ernesto Octavio López Ramírez (†)
}

\begin{abstract}
We describe an innovative approach to evaluate learning from a cognitive vision. Functionality of a constructive-chronometric evaluation model to measure academic learning is illustrated across several domains of knowledge. This evaluation model provides a wide range of indicators on cognitive characteristics of knowledge acquired throughout academic learning. The studies' evidence suggests that the evaluation model proposed here is effective in the cognitive measurement of academic learning. The implementation of cognitive science assessment tools in the field of education can be very useful to broaden our understanding of the knowledge acquisition process. These and other implications for the evaluation of student learning are discussed in this paper.
\end{abstract}

Keywords: digital learning evaluation; cognitive science; computational simulation; semantic priming; natural semantic network.

Citation: Morales Martínez, G. E., López Pérez, R. M., García Collantes, Á. y López Ramírez, E. O. (2020). Constructive-chronometric evaluation as a tool to evaluate online and face-to-face learning. Tecnología, Ciencia y Educación, 15, 105-124. 


\section{Sumario}

1. Introducción

2. El modelo cognitivo de evaluación constructiva-cronométrica del aprendizaje

3. Metodología

4. Participantes

5. Materiales e instrumentos

6. Procedimiento

7. Resultados

8. Discusión

9. Conclusiones

Referencias bibliográficas

Nota: este trabajo ha sido realizado como parte del proyecto A1-S-31770, registrado en el Fondo Sectorial de Investigación para la Educación (SEP-CONACYT), dentro de la Convocatoria CB-2017-2018. La investigación ha sido llevada a cabo parcialmente con recursos del Programa de Apoyo a Proyectos de Investigación e Innovación Tecnológica (PAPIIT) de la Universidad Nacional Autónoma de México (TA400116). Nuestros agradecimientos a la Coordinación de Humanidades, a la Dirección de Servicios Tecnológicos de la Coordinación de Innovación y Desarrollo y a la Dirección General de Asuntos del Personal Académico (DGAPA) de la Universidad Nacional Autónoma de México por el apoyo recibido en algunos de los proyectos de investigación incluidos en el presente trabajo. 


\section{Introducción}

Con la introducción de las nuevas tecnologías al aula escolar, la visión sobre la enseñanza y el aprendizaje se ha transformado. Por ejemplo, los sistemas de instrucción han ido cambiando con el desarrollo e implementación de los recursos educativos computarizados, las plataformas virtuales de enseñanza y las aplicaciones móviles, entre otros avances tecnológicos. Mientras tanto, en el campo del aprendizaje,

En el campo del aprendizaje, comienza a emerger una nueva visión acerca de la naturaleza psicológica de la mente del estudiante comienza a emerger una nueva visión acerca de la naturaleza psicológica de la mente del estudiante. En la actualidad, los estudiantes del aula del siglo XXI buscan participar de su propio proceso de aprendizaje en una forma más activa. Ellos no solamente reciben materiales sobre el conocimiento que desean aprender, sino que también crean, construyen, intercambian conocimiento, ya sea en forma individual o colaborativa; es decir, un estudiante nativo de la era digital no solamente recibe la información, sino que busca innovar y crear diferentes maneras de apropiarse y de generar el conocimiento.

Considerando estos cambios vertiginosos que caracterizan la naciente aula del siglo XXI, también surge la necesidad de reconceptualizar e innovar las herramientas del campo de la evaluación del aprendizaje. Aunque a lo largo de la historia, el concepto de «evaluación» se ha ido transformando debido a las necesidades y a los avances de cada contexto social, político y científico, sus medios no han variado mucho. Por ejemplo, en China, en el siglo XVII, se evaluaban las capacidades de los candidatos para algún puesto en el imperio a través de la tarea de memorizar 400.000 caracteres confusionistas (Gordon y Rajagopalan 2016). Esta primera visión resaltaba la idea de medir el conocimiento del participante para poder compararlo con otros, haciendo hincapié en la capacidad para recordar cantidades masivas de información. Posteriormente, durante el siglo XVIII, Farish propuso una innovación al establecer un sistema de calificación, otorgando marcas cuantitativas dependiendo del desempeño del estudiante. Esta idea reflejaba el interés de su época por demostrar la productividad educativa que estaba inspirada en el modelo industrial (Stufflebeam, Madaus y Kellaghan, 2002), y esto contribuyó al establecimiento de los parámetros de comparación.

Así, incorporando las premisas básicas de las propuestas anteriores, el sistema educativo integraba dos características a su modelo de evaluación del aprendizaje que prevalecen hasta la actualidad. La primera se refiere a la necesidad de crear instrumentos que midan el conocimiento del aprendiz, y la segunda, a la extracción de indicadores con valor numérico que 
describan el desempeño del estudiante. La influencia de estas dos características de la evaluación se puede observar en los medios e instrumentos para medir el aprendizaje que son utilizados en las aulas modernas (presenciales o virtuales).

Con respecto a lo anterior, es honesto reconocer que aunque instrumentos como los exámenes, los trabajos escolares y otro tipo de productos para medir el aprendizaje no son necesariamente aceptados por todos los protagonistas de la educación, estos han sido herramientas útiles porque brindan información sobre el aprendizaje del estudiante (por ejemplo, aquellos aspectos que el alumno no domina por completo) y permiten obtener un parámetro de comparación en términos de desempeño que, utilizado en forma constructiva, puede ser de ayuda para el maestro y para el estudiante (Brown, Roediger III y McDaniel, 2014). Sin embargo, dado los cambios contextuales, sociales, tecnológicos e incluso culturales y científicos, existen debates acerca de si estas herramientas pueden seguir aplicándose y utilizándose de la forma en que se ha hecho, ya que las herramientas de evaluación existentes no cubren todas las necesidades de las nuevas formas de conceptualizar la evaluación, el aprendizaje académico y la educación (Calenda y Tammaro, 2015; Morales-Martínez, López-Ramírez, García-Duran y Urdiales-Ibarra, 2018).

En la actualidad, el término «evaluación» se puede entender de muy diversas maneras (Corrigan, Buntting, Jones y Gunstone, 2013); por ejemplo, esta se puede entender como un medio de construcción de parámetros que ayudan a determinar si las metas planteadas se han cumplido (Shute y Becker, 2010). La evaluación también puede ser vista como un monitoreo del proceso de aprendizaje (Fitzgerald y Gunstone, 2013), o bien como un recurso de retroalimentación para mejorar áreas de oportunidad (Tillema, 2009).

Además, el Comité de Estándares para la Evaluación Educativa (en inglés, the Joint Committee on Standards for Educational Evaluation) ha propuesto criterios para que la evaluación del aprendizaje actual sea considerada como una herramienta recomendable y valiosa. En general, esta debe mostrar utilidad, factibilidad, exactitud y ser ética (Stufflebeam et al., 2002).

En términos de utilidad, la evaluación cumple con este criterio cuando contribuye a cubrir las necesidades educativas locales, institucionales, nacionales e incluso internacionales. A este respecto, las instituciones nacionales e internacionales utilizan la evaluación para fines diagnósticos, ya sea de tipo sumativo (Muldoon y Lee, 2007), con indicadores formales o estandarizados (Gipps, 1994; Madaus, Haney y Kreitzer, 2002); o bien como un instrumento formativo para determinar cuál será el punto de partida, dado el estado de aprendizaje en el que se encuentra el estudiante (Joughin, 2009); o para mejorar un programa educativo, dado lo observado en el proceso de adquisición del conocimiento (Bell y Bronwen, 2002; Sanders y Horn, 1995). 
Aunque la conceptualización de la evaluación se ha diversificado y comienza a plantearse la necesidad de introducir nuevos modelos de evaluación más acordes a los actuales modelos de enseñanza-aprendizaje, aún queda mucho que hacer en el campo de la instrumentación. Uno de los retos del presente siglo es la creación e innovación de nuevos medios de valoración del aprendizaje que vayan más allá del encapsulamiento digital de las herramientas de evaluación que están disponibles desde el siglo pasado (exámenes, portafolios, rúbricas, etc.) (Morales-Martínez, López-Ramírez y López-González, 2015).

Con respecto a lo anterior, en este artículo se describe una propuesta para complementar de una forma innovadora los instrumentos de evaluación del aprendizaje ya existentes. Aquí se propone y se ilustra el uso integrado de las herramientas provenientes de la ciencia cognitiva relacionada con la memoria y el aprendizaje humano, la aplicación de avances de la ciencia computacional y la tecnología para innovar y complementar los sistemas de evaluación del aprendizaje académico en niveles de educación superior. En particular, se ilustran algunos de los resultados obtenidos con el uso de un sistema cognitivo de orientación constructiva/cronométrica llamado
Aquí se propone y se ilustra el uso integrado de las herramientas provenientes de la ciencia cognitiva relacionada con la memoria y el aprendizaje humano, la aplicación de avances de la ciencia computacional y la tecnología para innovar y complementar los sistemas de evaluación del aprendizaje académico. EVCOG permite al maestro empoderar sus juicios de valor sobre el conocimiento internalizado en el estudiante «EVCOG» (evaluador cognitivo), que permite al maestro empoderar sus juicios de valor sobre el conocimiento internalizado en el estudiante (López, Morales, Hedlefs, González y Moreno, 2015; Morales, López, Hedlefs y González, 2014; Morales-Martínez y López-Ramírez, 2016; Morales-Martínez y SantosAlcántara, 2015; Morales-Martínez et al., 2015; Morales-Martínez, López-Ramírez y Velasco-Moreno, 2016; Morales-Martínez, López Ramírez et al., 2018; Morales-Martínez, López-Ramírez, Castro-Campos, Villarreal-Treviño y Gonzales-Trujillo, 2017; MoralesMartínez, Mezquita-Hoyos, González-Trujillo, López-Ramírez y García-Durán , 2018).

\section{El modelo cognitivo de evaluación constructiva-cronomé- trica del aprendizaje}

La psicología cognitiva asume que todo proceso cognitivo toma un tiempo que lo tipifica, incluso el aprendizaje (Lachman, Lachman y Butterfield, 1979). En particular, cuando un nuevo esquema de conocimiento es adquirido o uno ya existente es modificado durante el aprendizaje, se genera una actividad cognitiva que puede ser tipificada por su naturaleza temporal (huella temporal). Entonces, midiendo la presencia temporal de un esquema durante el aprendizaje, así como el efecto de este en el procesamiento de la información 
semánticamente relacionada, es posible la especificación cognitiva de varios aspectos relacionados a la adquisición de nuevo conocimiento a largo plazo (López y Theios, 1992).

En el modelo constructivo-cronométrico se asume que, al inicio de un curso, los estudiantes no poseen relación semántica entre los conceptos del esquema del contenido del curso, por lo tanto, los tiempos de reconocimiento de estos conceptos en estudios de facilitación semántica deben ser diferentes a los que se presentarán al final del curso cuando ya están integrados al esquema aprendido. También, sus tiempos de reconocimiento serán diferentes de los presentados en el reconocimiento de otros conceptos que no están relacionados con el curso y que no son el objetivo del contenido curricular. A este fenómeno se le conoce como «facilitación esquemática» (López, 1996; López y Theios, 1992). Si una red neurocomputacional es entrenada exitosamente para reconocer esta diferencia temporal de reconocimiento conceptual, entonces dicha red neural puede discriminar con un alto porcentaje de exactitud entre estudiantes que sí integraron conocimiento en su memoria a largo plazo y aquellos que no lo integraron al final del curso (Morales-Martínez y LópezRamírez, 2016). Estos cuatro aspectos de la medición cognitiva del aprendizaje (temporalidad, estructura, organización y dinámica del esquema) son la base de la propuesta del modelo de evaluación constructiva-cronométrica y su instrumento de medición, un sistema computarizado llamado EVCOG (véase figura 1) (Morales-Martínez y López-Ramírez, 2016).

Un estudio típico sobre aprendizaje académico, utilizando el EVCOG, comprende dos fases. En la primera, se lleva a cabo la evaluación constructiva del conocimiento, que involucra la aplicación, al inicio y al final del curso, de estudios de representación mental del esquema aprendido a través de la técnica de las redes semánticas naturales (RSN) (véase el apartado «Metodología»), que, a diferencia de otras técnicas de redes semánticas artificiales o idiosincráticas (por ejemplo, Itoyama, Nitta y Fujiki, 2007), realiza el análisis semántico de definiciones conceptuales obtenidas del propio estudiante, lo que permite la observación de los cambios conceptuales en la memoria a largo plazo, dado un aprendizaje.

Lo anterior se realiza a través de la inspección visual de la representación gráfica de las definiciones conceptuales del estudiante (véase figura 1 a) y b). Posteriormente, los resultados de las RSN (véase figura 1 a) y b) permiten obtener una matriz de pesos de conectividad entre los conceptos del esquema de conocimiento bajo análisis. Esto permite simular la emergencia de esquemas de nuevo conocimiento adquirido usando una red neural artificial (véase figura 1 d) con el objetivo de observar el comportamiento esquemático, representado visualmente con una gráfica de superficie de valores de matriz (véase figura $1 \mathrm{c}$ ). Lo anterior es congruente con los avances teóricos experimentales en el estudio de la memoria humana, que apoyan la idea de que un esquema de conocimiento no es una entidad cognitiva existente en la memoria, sino más bien que emerge conforme la situación lo requiera (Rogers y McClelland, 2004; Rumelhart, Smolensky, McClelland y Hinton, 1986).

Los resultados de la evaluación constructiva sientan las bases para la segunda fase de los estudios con el EVCOG; esto es, la evaluación cronométrica del aprendizaje. Para ello, 
utilizando los datos de las RSN, más los resultados obtenidos del comportamiento del esquema simulado, es posible seleccionar conceptos relacionados por el esquema adquirido en un curso, que serán usados para evaluar si un estudiante integró nueva información en su memoria a largo plazo a través de estudios de facilitación semántica (véase el apartado «Metodología»), que permiten el análisis de los tiempos de reconocimiento de estos conceptos (véase figura 1 e). Dichos tiempos de reconocimiento conceptual le permiten a un clasificador neural (véase figura $1 \mathrm{f}$ ) distinguir entre estudiantes que logran consolidar el aprendizaje a largo plazo y aquellos estudiantes que retienen el conocimiento solo por un corto plazo o que no logran integrar información en su memoria.

Figura 1. Parte de la interfaz del sistema EVCOG para ilustrar cada una de las etapas del modelo de evaluación cognitiva constructiva-cronométrica

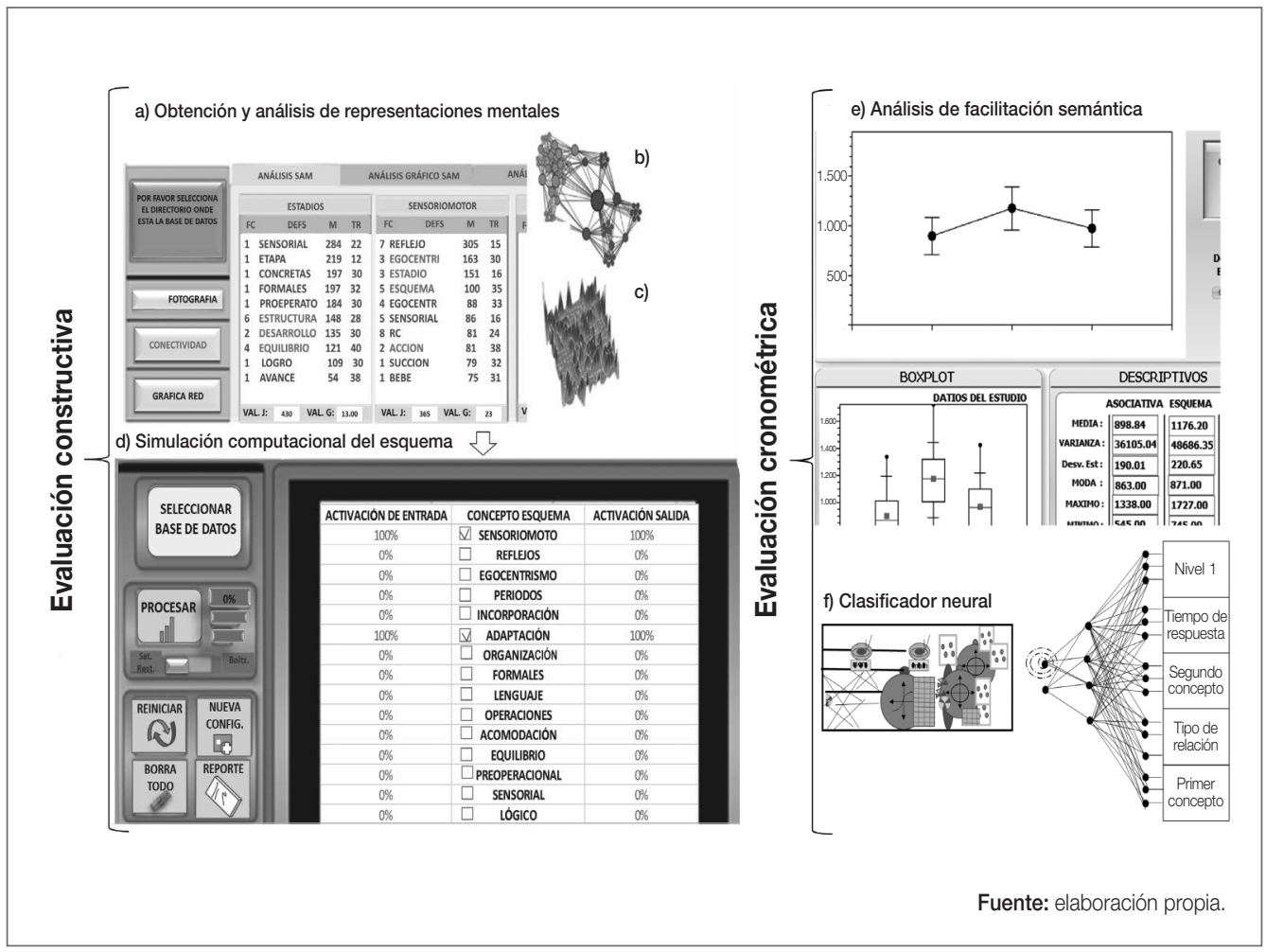

EI EVCOG ha sido implementado en cursos presenciales y en línea para medir el aprendizaje de estudiantes a nivel medio superior y superior en diferentes dominios del conocimiento (psicología, matemáticas, biología, etc.) (por ejemplo, véanse González, López y Morales, 2013; López et al., 2015; Morales et al., 2014; Morales-Martínez y López-Ramírez, 2016; Morales-Martínez y Santos-Alcántara, 2015; Morales-Martínez et al., 2015; Morales- 
Martínez et al., 2016; Morales-Martínez, LópezRamírez et al., 2018; Morales-Martínez, MezquitaHoyos et al., 2018; Urdiales-Ibarra, López-Ramírez, Castro-Campos, Villarreal-Treviño y Carrillo-Colón, 2018). Algunos de estos estudios son retomados, reanalizados y descritos en el siguiente apartado para ilustrar la utilidad del EVCOG como una herramienta alternativa en la evaluación cognitiva del aprendizaje académico.

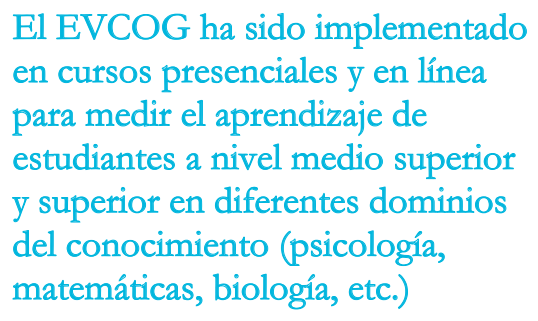

\section{Metodología}

Para ejemplificar el uso de la evaluación cognitiva constructiva-cronométrica del aprendizaje a través del sistema EVCOG, se describen tres estudios. El objetivo es ilustrar de forma general la efectividad del uso combinado de la tecnología cognitiva, específicamente el sistema EVCOG, con otras herramientas tecnológicas disponibles, para el análisis del efecto que tiene la adquisición y organización de nuevo conocimiento sobre el reconocimiento de palabras relacionadas por un esquema adquirido en cursos de diferentes dominios del conocimiento.

Los dos primeros estudios evalúan el aprendizaje en dos campos del conocimiento (la teoría sicogenética constructivista y la teoría computacional de la mente) impartidos en la carrera de Psicología. En el tercer estudio se midió el aprendizaje en un campo de conocimiento impartido en la carrera de Ingeniería (usabilidad computacional). Dado que el método para cada estudio fue el mismo, a continuación, se hace una descripción general del mismo.

\section{Participantes}

Para la evaluación de los dominios de conocimiento en el campo de la psicología, se requirió la participación de una muestra de 44 estudiantes (33 mujeres y 11 hombres) que cursaban la materia de Teoría Psicogenética Constructivista. Sus edades oscilaron entre los 18 y los 23 años. Además, participaron 49 estudiantes (37 mujeres y 12 hombres) de la materia Teoría Computacional de la Mente. Sus edades oscilaron entre los 17 y los 21 años. Del campo de la ingeniería participó una muestra de 53 estudiantes (22 mujeres y 31 hombres) que cursaban la materia de Usabilidad Computacional. Sus edades oscilaron entre los 20 y los 27 años. Todos los participantes fueron voluntarios y recibieron puntos por su colaboración. 


\section{Materiales e instrumentos}

Para los estudios de representación mental, se seleccionaron 10 conceptos de cada dominio del conocimiento evaluado en cada materia. Para el curso Teoría Psicogenética Constructivista se utilizaron los siguientes conceptos objetivo: «estadios», «sensoriomotor», «preoperatoria», «concretas», «formales», «adaptación», «asimilación», «acomodación», «equilibrio» y «esquema». Para el curso Teoría Computacional de la Mente se evaluaron los siguientes objetivos: «mente», «computación», «Von Neumann», «procesamiento humano de información», «mente computacional», «máquina de Turing», «conexionismo», «memoria», «memoria de trabajo» y «memoria a largo plazo». Y para el curso Usabilidad Computacional, los 10 conceptos objetivos fueron los siguientes: «diseñador», «evaluación», «implementación», «interacción humano computadora», «análisis de registro», «interfaz», «lanzamiento», «programador», «prototipo» y «usabilidad».

Cada concepto objetivo fue seleccionado cuidadosamente por el maestro responsable de impartir la materia, siguiendo el procedimiento del protocolo para la recolección de conceptos objetivo y definidores centrales y diferidos (PRECODECD) de Morales (2015). Utilizando el PRECODECD también se extrajeron los conceptos facilitadores y los objetivos utilizados en cada estudio cronométrico. Por ejemplo, para la materia Teoría Psicogenética Constructivista se incluyeron en el diseño del estudio conceptos como "estadio», «sensoriomotor», «epistemología», «símbolo», «estructura», etc. Para la materia Teoría Computacional de la Mente se eligieron conceptos como «procesos», «lenguaje», «probabilidad», «procesador» y «metáfora». Y para la materia Usabilidad Computacional, se seleccionaron conceptos como «interfaz», «evaluar», «código», «sistema», etc.

Para la construcción de los instrumentos constructivos (RSN) y cronométricos (facilitación semántica [FACSEM]) se utilizó el sistema EVCOG, que es un software que permite configurar, aplicar y analizar los datos obtenidos de los estudios de evaluación constructiva-cronométrica.

\section{Procedimiento}

En cada curso, el aprendizaje se evaluó con el mismo procedimiento. Primero, se requirió el consentimiento informado de cada participante. Posteriormente, al inicio y al final de cada ciclo escolar, cada muestra participó en una serie de estudios en ciencia cognitiva siguiendo las primeras tres fases del sistema de evaluación constructiva-cronométrica con el sistema EVCOG (véase figura 1). Es decir, para cada materia evaluada se aplicó primero un estudio de representación del conocimiento, después se realizó una simulación computacional del esquema de conocimiento y, finalmente, un estudio de cronometría mental. La cuarta fase se refiere al uso de un neuroclasificador de tres capaz con retropropagación del error y consiste en la realización de simulaciones que permitirán determinar a qué grupo de aprendizaje (a corto o largo plazo) pertenece el patrón de respuesta del estudiante. Sin embargo, en este artículo no se describe esta fase dado lo extenso del tema. 
En el primer estudio se exploró la representación mental que tenían los estudiantes sobre el esquema de conocimiento estudiado en el curso. Para ello, se requirió a los participantes realizar una tarea definicional, basada en la técnica de las RSN (Figueroa, González y Solís, 1976, 1981; López, 1996; López y Theios, 1992), que consistió en definir 10 conceptos objetivo o centrales al esquema de conocimiento. Estos conceptos fueron presentados a través del programa de computadora EVCOG. La tarea cognitiva consistía en definir uno a uno cada concepto objetivo con conceptos definidores (verbos, sustantivos, pronombres y adjetivos) y después ponderar cada definidor dado utilizando una escala de 1 a 10, donde 1 significaba que el definidor dado estaba poco relacionado o definía poco el objetivo, mientras que 10 significaba que se relacionaba o definía bastante bien el objetivo.

Con los resultados obtenidos del primer estudio, se realizaron simulaciones computacionales para explorar el comportamiento del esquema. El EVCOG permitió obtener la matriz de conectividad, con la cual fue posible implementar la red neural (del tipo de satisfacción de restricciones [véase Rumelhart et al., 1986]) que fue capaz de simular el esquema de conocimiento emergente y permitió determinar los conceptos integrados en el esquema de conocimiento aprendido, así como observar el comportamiento esquemático de cada dominio de conocimiento evaluado al inicio y al final del ciclo escolar.

En el tercer estudio, se exploró la consolidación del esquema aprendido en la memoria de los estudiantes a través de un estudio de facilitación semántica, el cual consistía en la presentación de pares de palabras facilitador-objetivo que podían estar relacionadas con el esquema de conocimiento evaluado, o bien podían estar relacionadas a través de alguna asociación o no tener relación alguna. Estos pares eran presentados al azar en la pantalla de una computadora utilizando el EVCOG. La tarea experimental consistía en leer cada palabra en la mente y después clasificar la última palabra (objetivo) de cada par como bien escrita o mal escrita. Aquí, se midió la respuesta dada y el tiempo de reacción que el participante tardaba en categorizar cada palabra.

\section{Resultados}

Los datos obtenidos de los estudios de representación mental y de las simulaciones computacionales fueron analizados desde la perspectiva de la evaluación cognitiva-constructiva. Primero, utilizando el EVCOG se llevó a cabo el análisis representacional de los datos obtenidos de estudiantes que cursaban la materia Teoría Computacional de la Mente. Para ello, se identificaron los 10 grupos de definición conceptual, o grupos SAM (semantic analysis of $M$ value), dados por los estudiantes antes y después del curso. Por cuestiones de espacio, en la figura 2 se muestra la captura de la pantalla de computadora del sistema software EVCOG con solo 3 de los 10 grupos SAM obtenidos al inicio del semestre. Los resultados detallados de este análisis de RSN se pueden ver en la tesis de García (2018) o en la obra de Morales (2019). 
Además, se realizó un análisis con GEPHI, que es un software de libre uso para explorar y manipular redes (Bastian, Heymann y Jacomy, 2009). Para el análisis se utilizó la matriz de conectividad extraída con el EVCOG. Esto permitió llevar a cabo un análisis visual de los grupos conceptuales de la RSN de la materia Teoría Computacional de la Mente para obtener una representación visual de la red semántica antes y después del curso que se muestra en la figura 2. En la figura 2 b) se muestra que, al inicio del curso, los estudiantes de esta materia poseen información conceptual que no está integrada al resto de información que poseen antes del aprendizaje (señalados por el cuadro en línea discontinua). Las diferencias de cantidad y organización conceptual entre el inicio y final del curso se hacen evidentes a través de un análisis visual cualitativo de la representación visual de la red conceptual obtenida. Obsérvense en la figura 2 c) los cambios en la estructura y organización conceptual del esquema de conocimiento que se producen después del curso. Aquí, se asume que estos son debidos al aprendizaje que los estudiantes obtuvieron durante su ciclo escolar.

Figura 2. Representación visual de los resultados de redes semánticas utilizando el sistema EVCOG y el sistema GEPHI

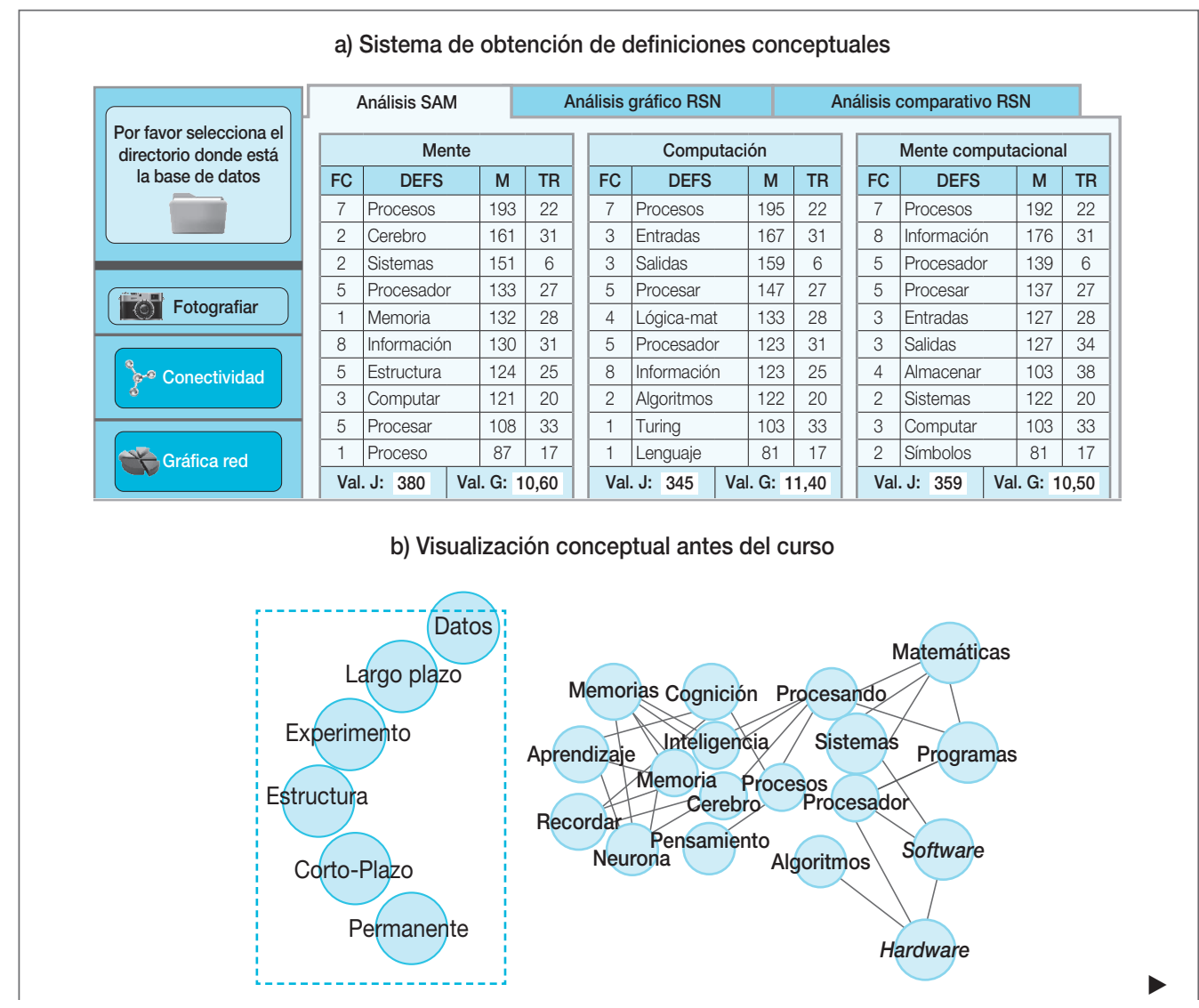


c) Visualización conceptual después del curso

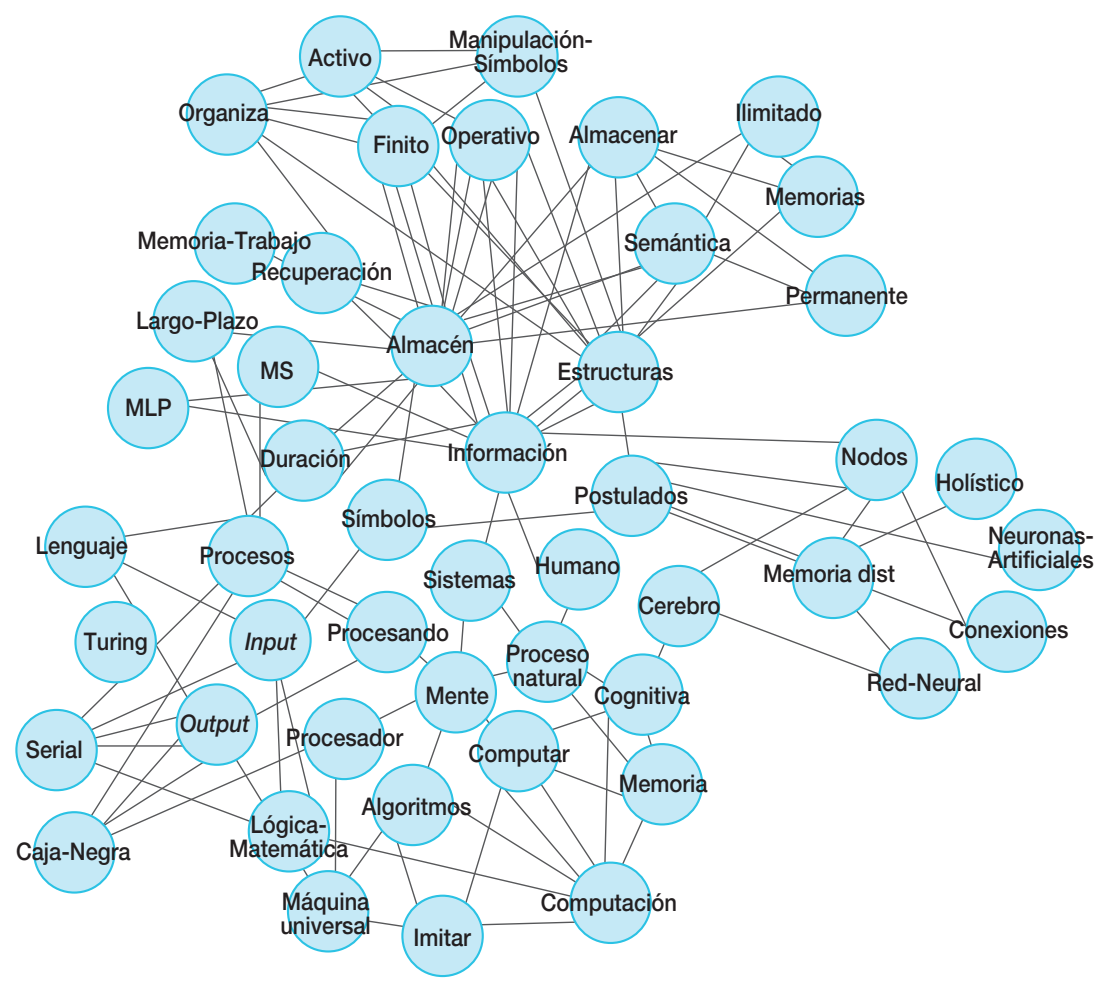

Nota: en la imagen a) de esta figura se muestra una captura de pantalla de los grupos SAM o definiciones conceptuales obtenidas con el sistema de EVCOG para tres de los conceptos objetivo; además, se muestra la representación visual de la conectividad conceptual de los 10 grupos SAM, antes del curso (véase b) y después del curso (véase c), obtenida del sistema GEPHI.

Fuente: la imagen a) ha sido extraída de la tesis de licenciatura inédita de García (2018, p. 85). Derechos reservados (2018) por la Universidad Pedagógica Nacional (Ajusco). Imagen adaptada con permiso de su autor. Las imágenes b) y c) son de elaboración propia.

Después del análisis de las RSN y de la inspección visual, se extrajo la matriz de conectividad entre los conceptos definidores obtenidos utilizando el EVCOG. Posteriormente, fue posible simular computacionalmente el comportamiento del esquema adquirido en un curso. La figura 3 muestra una captura del simulador computacional EVCOG para el esquema del curso Teoría Computacional de la Mente. Nótese que activando uno o varios conceptos es posible ver patrones de coactivación de salida que reflejan el efecto del esquema implícito en los pesos de conectividad entre conceptos. Esto permite identificar qué conceptos permiten mayor influencia del esquema (relación semántica de esquema) al ser activados. 
Figura 3. La activación del concepto "símbolos» coactiva conceptos relacionados con el esquema de conocimiento del curso Teoría Computacional de la Mente (al final del curso)

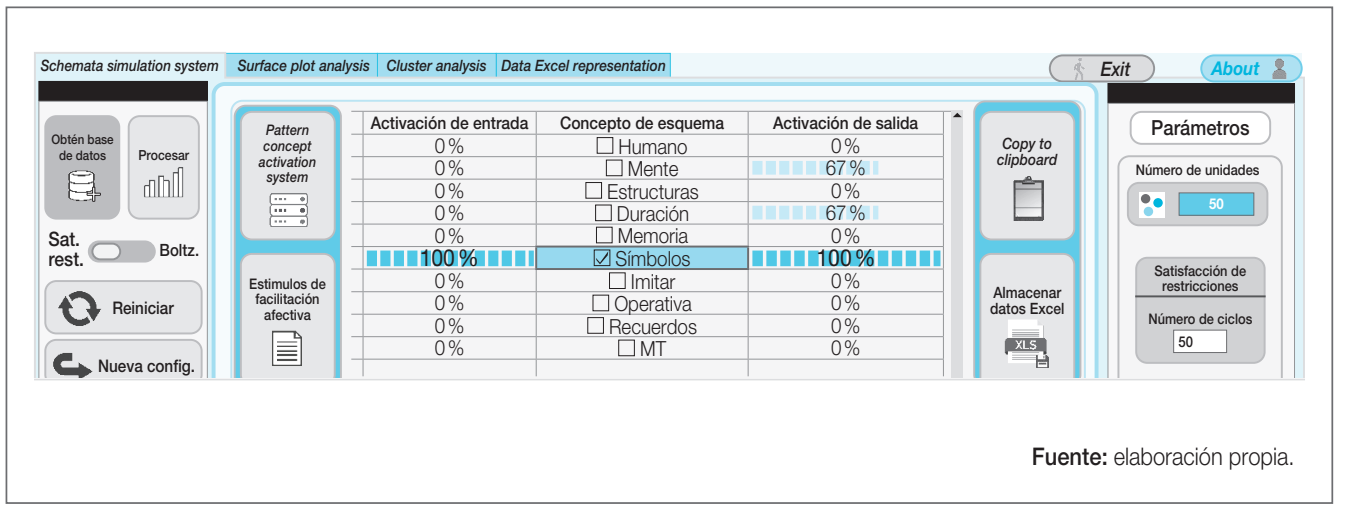

Estos dos tipos de análisis también fueron llevados a cabo en las otras dos materias. Dichos análisis permitieron determinar los conceptos con mayor peso semántico y mayor activación. Este material permitirá construir los estudios de facilitación semántica en cada uno de los campos de conocimiento evaluado, considerando mayores controles experimentales con respecto a la selección de las entradas o estímulos en estudios posteriores.

Con respecto al análisis de los datos obtenidos durante la evaluación cronométrica, los resultados señalan que, al final del ciclo escolar, los estudiantes de los diferentes cursos que obtuvieron calificaciones aptas reconocen, de forma significativamente más rápida, las palabras relacionadas por el esquema de conocimiento del curso en comparación con el tiempo que les llevaba el reconocimiento de palabras relacionadas esquemáticamente al inicio del curso.

La figura 4 muestra los tiempos de reconocimiento de los participantes de las palabras relacionadas por los diferentes esquemas de conocimiento consideradas en la evaluación cronométrica de cada curso (para mayor detalle de estos hallazgos se puede consultar Morales et al., 2016; García, 2018; Morales-Martínez y López-Ramírez, 2016).

Resultados similares fueron obtenidos en el seguimiento de estudiantes a través de dos semestres consecutivos, en los que cursaron la materia Usabilidad Computacional secuenciada en dos niveles de profundidad. En este estudio, los participantes mostraron tiempos de reconocimiento de palabras esquemáticas significativamente menores al inicio del curso, comparados con los obtenidos al final del mismo (para mayor detalle de estos hallazgos se puede consultar Morales-Martínez y López-Ramírez, 2016). Y como se puede apreciar en la figura 5, la diferencia de tiempo fue de menor magnitud en los estudiantes del segundo semestre que en los del primero. 
Figura 4. Tiempos de reconocimiento de palabras relacionadas por el esquema de conocimiento aprendido antes y después de un curso

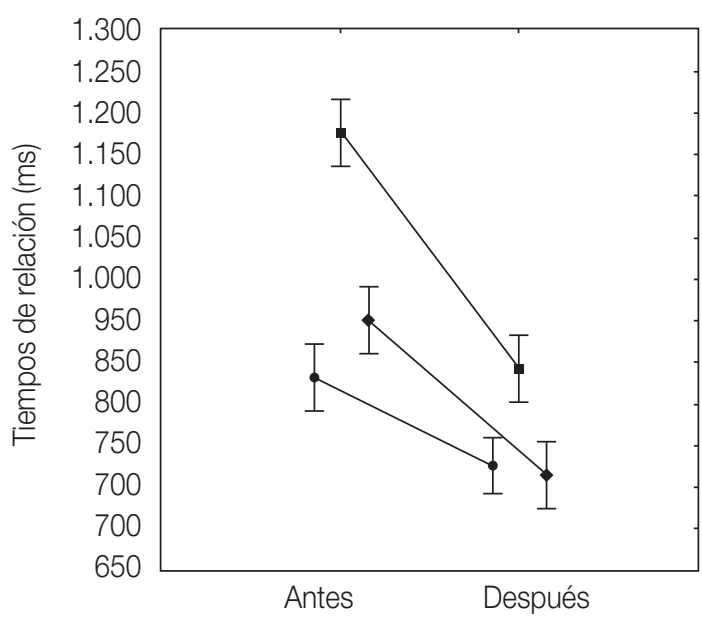

\section{Cursos}

Ð- Teoría psicogenética de Piaget

三 Teoría computacional de la mente

$\Xi$ Usabilidad computacional

Pares de palabras con relación esquemática

Fuente: elaboración propia.

Figura 5. Latencias de tiempo que utilizaron los participantes del primer y del segundo semestre de Ingeniería para reconocer palabras con relación esquemática antes y después del curso de usabilidad computacional

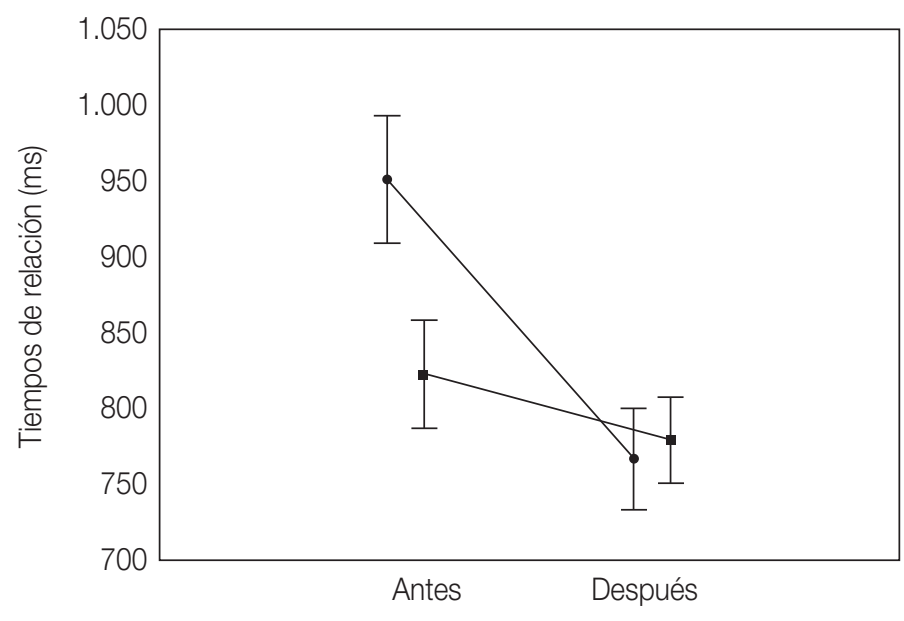

Semestre

$\exists$ Primero

$\exists$ Segundo

Pares de palabras con relación esquemática

Fuente: elaboración propia 


\section{Discusión}

El objetivo de los estudios aquí presentados ha sido ilustrar el modo en que, utilizando herramientas provenientes de la ciencia cognitiva, es posible innovar o crear instrumentos, e incluso modelos de evaluación, que pueden contribuir con indicadores cognitivos del aprendizaje, que, sumados a los indicadores ya utilizados, empoderan al maestro, al estudiante e incluso a la institución para tomar decisiones basadas en una mayor comprensión del comportamiento y del mecanismo cognitivo de la mente del estudiante durante su proceso de aprendizaje.

En general, los resultados mostraron que el uso de sistemas como el EVCOG es de utilidad para analizar aspectos cognitivos del aprendizaje. Por ejemplo, la evaluación constructiva realizada en los estudios aquí descritos brindó información acerca de cómo los esquemas tienen propiedades estructurales y organizativas diferentes, dado el estado de aprendizaje en el que se encuentra el estudiante (véase figura 2). Se han obtenido resultados similares en otros estudios (por ejemplo, Morales-Martínez y et al., 2016) que muestran cómo los estudiantes relacionan y arreglan los conceptos de forma diferente al inicio y al final del curso.

Los estudios de simulación computacional mostraron ser útiles para explorar el comportamiento del esquema. En este caso, con el software EVCOG fue posible identificar el grado de activación y coactivación de los conceptos del esquema (véase figura 3); es decir, provee información sobre la dinámica del esquema que es valiosa para contar con mayores controles en la selección de los estímulos que se van a utilizar en los diseños de los estudios cronométricos.

Con respecto a los estudios de cronometría mental (en este caso, el uso de la facilitación semántica), mostraron ser sensibles a cambios en la consolidación del esquema aprendido. Por ejemplo, los resultados de los estudios (véanse figuras 4 y 5) sugieren que los tiempos de reacción pueden ser considerados como manifestaciones cognitivas automáticas que no están contraladas de forma consciente por el estudiante y pueden reflejar aspectos temporales de la activación conceptual de la información aprendida e integrada en el lexicón del estudiante (por ejemplo, McNamara, 2005). En este caso, los tiempos de reacción consumidos para reconocer conceptos integrados por el esquema son significativamente menores al final del curso si se los compara con los presentados al inicio del mismo. Esto sugiere que hubo un cambio en la huella temporal de reconocimiento, dada la integración y consolidación de los conceptos esquemáticos en la memoria del estudiante (véanse figuras 4 y 5).

Las técnicas cronométricas pueden ser muy útiles para determinar si existe integración de los conceptos del curso en un esquema en la memoria del estudiante. Y, si son utilizadas en forma conjunta con la evaluación constructiva, pueden convertirse en una útil herramienta de intervención educativa. 


\section{Conclusiones}

En general, los resultados indican que sistemas como el EVCOG, en conjunto con el uso de otras tecnologías ya disponibles, como el sistema GEPHI (véase Bastian et al., 2009), pueden proveer indicadores cuantitativos y cualitativos de la organización y de la estructura del esquema de conocimiento bajo estudio que serán útiles para comprender la realidad cognitiva que un estudiante experimenta durante su proceso de aprendizaje (Bersano-Méndez, Schaefer y Bustos-Jiménez, 2012; Morales-Martínez y Santos-Alcántara, 2015).

El modelo de evaluación constructiva-cronométrica del conocimiento propuesto aquí pretende contribuir con el desarrollo de nuevas tecnologías para llevar a cabo la evaluación formativa del aprendizaje, donde el objetivo es observar el proceso cognitivo de construcción de conocimiento (Muldoon y Lee, 2007) para crear mejores condiciones que favorezcan el desarrollo académico del estudiante. Por ejemplo, el modelo aquí propuesto ofrece la posibilidad de obtener información del estado de aprendizaje de los estudiantes en diferentes momentos del ciclo escolar, lo que brinda herramientas para tomar decisiones sobre el proceso de aprendizaje de los estudiantes. Es decir, esta propuesta contribuye a la evaluación formativa del aprendizaje, donde el objetivo es observar el proceso cognitivo de construcción de conocimiento (Muldoon y Lee, 2007) y retroalimentar a los protagonistas de la educación a través de una herramienta digital llamada EVCOG que empodera a investigadores y maestros en el diseño y en el análisis de estudios constructivos-cronométricos y que puede ser aplicada de manera presencial y en línea.

Finalmente, el modelo de evaluación constructiva-cronométrica, en conjunto con su herramienta EVCOG, pueden permitir la exploración de nuevas áreas del proceso de aprendizaje que nos permitan ampliar nuestra comprensión de la naturaleza cognitiva de este proceso. Por ejemplo, nuevas aplicaciones de esta visión se están extendiendo a la exploración de los mecanismos cognitivos del escaneo visual de textos (véase Morales-Martínez, Mezquita-Hoyos et al., 2018) y comienzan a brindar información sobre los procesos cognitivos que subyacen a la apropiación del conocimiento en la mente de los estudiantes.

\section{Referencias bibliográficas}

Bastian, M., Heymann, S. y Jacomy, M. (2009). Gephi: An Open Source Software for Exploring and Manipulating Networks. Association for the Advancement of Artificial Intelligence. Recuperado de <https://gephi.org/publica tions/gephi-bastian-feb09.pdf> (consultado el 8 de agosto de 2019).
Bell, B. y Bronwen C. (2002). Formative Assessment and Science Education. Nueva York: Science \& Technology Education Library/ Kluwer academic Publisher.

Bersano-Méndez, N. I., Schaeffer, S. E. y BustosJiménez, J. (2012). Metrics and models for 
social networks. En A. Abraham y A. E. Hassanien (Eds.), Computational Social Networks: Tools, Perspectives and Applications (pp. 115-142). Londres: Springer Verlag. doi: 10.1007/978-1-4471-4048-1.

Brown, P. C., Roediger III, H. L. y McDaniel, M. A. (2014). Apréndetelo: la ciencia del aprendizaje exitoso. México: Paidós.

Calenda, M. y Tammaro, R. (2015). The assessment of learning: from competence to new evaluation. Procedia-Social and Behavioral Sciences, 174, 3.885-3.892. doi: 10.1016/j.sbspro.2015.01.1129.

Corrigan, D., Buntting, C., Jones, A. y Gunstone, R. (2013). Valuing assessment in science education: an introductory framework. En D. Corrigan, R. Gunstone y A. Jones (Eds.), Valuing Assessment in Science Education: Pedagogy, Curriculum, Policy (pp. 1-9). Dordrecht, Holanda: Springer. doi: 10.1007/978-94-007-6668-6.

Figueroa, J. G., González, E. G. y Solís, V. M. (1976). An approach to the problem of meaning: semantic networks. Journal of Psycholinguistic Research, 5(2), 107-115. doi: 10.1007/BF01067252.

Figueroa, J. G., González, E. G. y Solís, V. M. (1981). Una aproximación al problema del significado: las redes semánticas. Revista Latinoamericana de Psicología, 13(3), 447-458.

Fitzgerald, A. y Gunstone, R. (2013). Embedding assessment within primary school science: a case study. En D. Corrigan, R. Gunstone, R. y A. Jones, A. (Eds.), Valuing Assessment in Science Education: Pedagogy, Curriculum, Policy (pp. 307-324). Dordrecht, Holanda: Springer. doi: 10.1007/978-94-007-6668-6.

García, D. J. P. (2018). Evaluación del aprendizaje del esquema de conocimiento adquirido en una materia de psicología desde una perspectiva conexionista (Tesis de licenciatura inédita). Universidad Peda- gógica Nacional Plantel Ajusco. Licenciatura en Psicología Educativa. Ciudad de México, México.

Gipps, C. V. (1994). Beyond Testing: Towards a Theory of Educational Assessment. Londres: The Falmer Press.

González, C. J., López, E. O. y Morales, G. E. (2013). Evaluating moral schemata learning. International Journal of Advances in Psychology, 2(2), 130-136.

Gordon, E. W. y Rajagopalan, K. (2016). The Testing and Learning Revolution: The Future of Assessment in Education. Nueva York: Palgrave Macmillan. doi: 10. 1057/9781137519962.

Itoyama, K., Nitta, T. y Fujiki, T. (2007). On the relation between semantic network and association map for the assessment of class work. En M. Iskander (Ed.), Innovations in E-learning, Instruction Technology, Assessment, and Engineering Education (pp.199-204). Dordrecht, Holanda: Springer.

Joughin. G. (Ed.). (2009). Assessment, Learning and Judgement in Higher Education. Dordrecht, Holanda: Springer. doi: 10. 1007/978-1-4020-8905-3.

Lachman, R., Lachman, J. L. y Butterfield, E. C. (1979). Cognitive Psychology and Information Processing: An Introduction. Hillsdale, Nueva Jersey: Lawrence Erlbaum Associates.

López, E. O. (1996). Schematically Related Word Recognition. (Order No. 9613356). Available from ProQuest Dissertations \& Theses Global (304292488). Recuperado de <https://search.proquest.com/docview/ 304292488?accountid=14598> (consultado el 8 de septiembre de 2018).

López, R. E. O. y Theios, J. (1992). Semantic analyzer of schemata organization (SASO). Behavior Research Methods, Instruments, \& Computers, 24(2), 277-285. doi: 10.3758/BF03203508. 
López, E. O.y Theios, J. (1996). Single word schemata priming: a connectionist approach. 69th Annual Meeting of the Midwestern Psychological Association. Chicago, IL.

López, E. O., Morales, G. E., Hedlefs, I. y González, J. (2014). New empirical directions to evaluate online learning. International Journal of Advances in Psychology, 3(2), 40-47. doi: 10.14355/ijap.2014.0302.03.

López, R. E. O., Morales, M. G. E., Hedlefs, A. M. ${ }^{a}$ I., González, T. C. J. y Moreno, M. A. P. (2015). Nuevas direcciones empíricas en la investigación e innovación de tecnología educativa para la evaluación del aprendizaje en línea: una aproximación conexionista. Ciencia UANL, 18(71), 52-64.

Madaus, G. F., Haney, W. y Kreitzer, A. (2000). The role of testing in evaluations. En D. L. Stufflebeam, G. F. Madaus y T. Kellaghan T. (Eds.), Evaluation Models: Viewpoints on Educational and Human Services Evaluation (pp. 113-125). (2nd ed.). Nueva York: Kluwer Academic Publishers.

McNamara, T. P. (2005). Semantic Priming: Perspectives from Memory and Word Recognition. Nueva York: Psychology Press.

Morales, M. G. E. (2015). Protocolo para la recolección de conceptos objetivo y definidores centrales y diferidos (PRECODECD): un sistema de codificación de conceptos extraídos de las redes semánticas naturales. Manuscrito no publicado, Instituto e Investigaciones sobre la Universidad y la Educación, Universidad Nacional Autónoma de Nuevo León.

Morales, M. G. E. (2019). Sistema de evaluación cognitiva constructiva cronométrica del aprendizaje en línea. Manuscrito presentado para su publicación.

Morales, M. G. E., López, R. E. O., Hedlefs, A. M. ${ }^{a}$ I. y González, T. C. J. (2014). Recuperando el paso en la evaluación del aprendizaje en línea en la era digital: una aproximación conexionista. Revista Ingenierías, 17(65), 27-36.

Morales-Martínez, G. E. y López-Ramírez, E. O. (2016). Cognitive responsive e-assessment of constructive e-learning. Journal of e-Learning and Knowledge Society, 12(4), 39-49. doi: 10.20368/1971-8829/1187.

Morales-Martínez, G. E. y Santos-Alcántara, M. ${ }^{a}$ G. (2015). Alternative empirical directions to evaluate schemata organization and meaning. Advances in Social Sciences Research Journal, 2(9), 51-58. doi: 10.14738/assrj.29.1412.

Morales-Martínez, G. E., López-Ramírez, E. O. y López-González, A. E. (2015). New approaches to e-cognitive assessment of e-learning. International Journal for e-Learning Security (IJeLS), 5(2), 449-453. doi: 10.20533/ijels.2046.4568.2015.0057.

Morales-Martínez, G. E., López-Ramírez, E. O. y Velasco-Moreno, D. (2016). Alternative e-learning assessment by mutual constrain of responsive and constructive techniques of knowledge acquisition evaIuation. International Journal for Infonomics (IJI), 9(3), 1.195-1.200. doi: 10.20533/iji.17 42.4712.2016.0145.

Morales-Martínez, G. E., López-Ramírez, E. O., García-Durán, J. P. y Urdiales-Ibarra, M. ${ }^{2}$ E. (2018). Cognitive Constructive-Chronometric Techniques as a Tool for the E-Assessment of Learning. International Journal of Learning, Teaching and Educational Research, 17(2), 159-176. doi: 10.26803/ijlter.17.2.10.

Morales-Martínez, G. E., López-Ramírez, E. O., Castro-Campos, C., Villarreal-Treviño, M. ${ }^{a}$ G., y Gonzales-Trujillo, C. J. (2017). Cognitive analysis of meaning and acquired mental representations as an alternative measurement method technique to innovate e-assessment. European Journal of Educational Research, 6(4), 455-464. doi: 10. 12973/eu-jer.6.4.455. 
Morales-Martínez, G. E., Mezquita-Hoyos, Y. N., González-Trujillo, C. J., López-Ramírez, E. O. y García-Durán, P. J. (2018). Formative e-assessment of schema acquisition in the human lexicon as a tool in adaptive online instruction (pp. 69-88). En R. López-Ruiz (Ed.), From Natural to Artificial Intelligence: Algorithms and Application. Londres, Reino Unido: IntechOpen.

Muldoon, N. y Lee, C. (2007). Formative and summative assessment and the notion of constructive alignment. En S. Frankland (Ed.), Enhancing Teaching and Learning through Assessment (pp. 98-108). Dordrecht, Holanda: Springer.

Rogers, T. T. y McClelland, J. L. (2004). Semantic Cognition: A Parallel Distributed Processing Approach. Massachusetts, EE. UU.: MIT Press.

Rumelhart, D. E., Smolensky, P., McClelland, J. L., y Hinton, G. (1986). Schemata and sequential thought processes. En J. L. McClelland, D. E. Rumelhart y The PDP Research Group. Parallel Distributed Processing: Explorations in the Microstructure of Cognition (Vol. 2, pp. 7-57). Massachusetts, EE. UU.: MIT Press.

Sanders, L. W. y Horn, P. S. (1995). Educational assessment reassessed: the usefulness of standardized and alternative measures of student achievement. Education Policy Analysis Archives, 3(6), 1-15.

Shute, V. J. y Becker, B. J. (2010). Prelude: assessment for the 21st century. En V. J. Shute y B. J. Becker (Eds.), Innovative Assessment for the 21st Century (pp. 1-11). Nueva York, EE. UU.: Springer. doi: 10.1007/978-1-4419-6530-1.

Stufflebeam D. L., Madaus G. F. y Kellaghan T. (Eds.). (2002). Evaluation Models: Viewpoints on Educational and Human Services Evaluation (2nd ed.). Nueva York: Kluwer Academic Publishers.

Tillema, H. H. (2009). Assessment for learning to teach: appraisal of practice teaching lessons by mentors, supervisors, and student teachers. Journal of Teacher Education, 60(2), 155-167. doi: 10.1177/0022487108330551.

Urdiales-Ibarra, M. ${ }^{a}$ E., López-Ramírez, E. O., Castro-Campos, C., Villarreal-Treviño, M. ${ }^{a} \mathrm{G}$. y Carrillo-Colón, J. E. (2018). Biology schemata knowledge organization and meaning formation due to learning: a constructivechronometric approach to concept mapping usability. Creative Education, 9(16), 2.6932.706. doi: 10.4236/ce.2018.916203. 